\title{
APPLICATIONS OF ARTIFICIAL INTELLIGENCE IN CYBER SECURITY
}

This paper was downloaded from TechRxiv (https://www.techrxiv.org).

LICENSE

CC BY 4.0

SUBMISSION DATE / POSTED DATE

$12-10-2021 / 14-10-2021$

CITATION

Thorat, Arvind (2021): APPLICATIONS OF ARTIFICIAL INTELLIGENCE IN CYBER SECURITY. TechRxiv. Preprint. https://doi.org/10.36227/techrxiv.16789237.v1

$\mathrm{DOI}$

10.36227/techrxiv.16789237.v1 


\section{APPLICATIONS OF ARTIFICIAL INTELLIGENCE IN CYBER SECURITY}

\section{Author: - Arvind Thorat}

Kavikulguru institute of technology and science Ramtek, Maharashtra, India

\section{ABSTRACT}

One of the most difficult problems in cybersecurity is identifying harmful information. This issue affects all types of security technologies, from intrusion detection systems to malware detection and application security solutions. The conventional technique to detection is employing regular expressions to compare the incoming request or its vector with a collection of preset patterns or signatures. Some signatures are open-source, such as the Core Rule Set, while others are from commercial sources. Although widely used, using signatures to categories inputs is not particularly accurate. The issues emerge not just because the signatures are not updated frequently enough, but also because the signatures are not updated at all.

General pattern of Cyber System (components of privacy, security, threads and conflicts)

\section{ASSETS}

\section{Privacy}

Content

Identity

pseudonymity

Anonymity

\section{SECURITY}
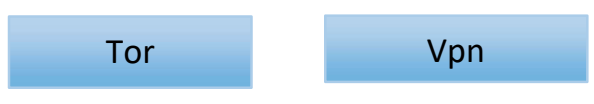

$$
\text { JonDonym }
$$

OpsecEncry

2FA Patching

\section{THREADS}

Vishing

Rats

\section{Phishing}

Viruses
Spying

Malware
Adware

Mass Surv
Spyware

Reg. Encryp
Rootkits

Exploit Kit 


\section{ADEVERSARIES}

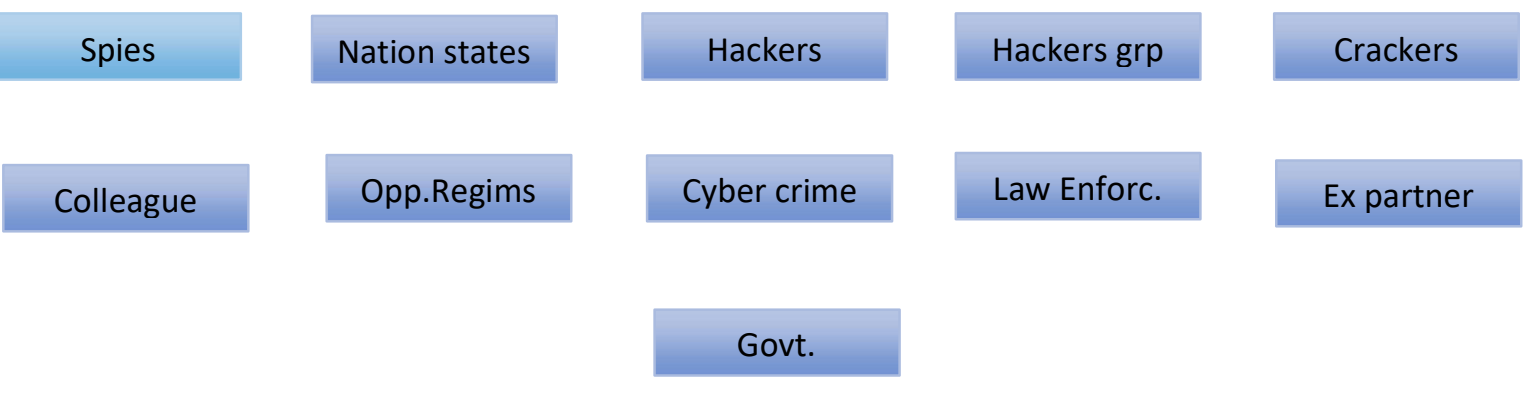

\section{Objective}

With the exponential rise in the propagation of threads associated with the daily dissemination of new malware, dealing effectively with these threads using just human analysis is almost impossible. It is necessary to create an algorithm that will allow us to automate the triage portion of the analysis.

As a consequence, cybersecurity professionals are needed in order to understand algorithm principles for okay their learning phase depend on the results and aim to be accomplished. Artificial intelligence is used very rarely for security purposes, as stated. But, with Al's aid, we can figure out the pattern of assault. To use it, we must figure out the threads.

Following are the possible methods in Al to detect the threads in Cyber Security

\section{Classification}

This is one of the main methods in the framework of cyber security Use to identify the types of similar attacks

\section{Clustering}

Clustering is distinguished from classification by the ability to automatically identify the classes to which the sample belong when information about classes is not available in advance. This methos is fundamentally important in malware analysis and forensic analysis.

\section{Predictive Analysis}

By exploiting NNS and DL. It is possible to identify threads as they occur. To this end a highly dynamic approach must be adopted which allows algorithm to optimize their learning capabilities automatically. 


\section{TYPES OF PROTECTION}

\section{New Protection}

The use of ML allows the implantation of highly sophisticated intrusion detection system. IDS Which are to be used in the network protection area.

\section{Endpoint Protection}

Threads such as ransomware can be adequately detected by adopting algorithm that learn these types of malwares. Thus, overcoming the limitations of traditional antivirus software.

\section{Application Security.}

Some of the most insidious types of web applications include server-side request forgery (SSRF) attacks, SQL injection, cross-site, Scripting (Xss) and Distributed Danial service attacks. These are all types of threads that can be adequately countered by using Al and ML tools and Algorithm.

\section{Suspects User behavior}

Identifying attempts at fraud or compromising applications by malicious users at the very Moment they occur in one of the emergent areas of applications of DL (Deep learning).

\section{METHODOLOGY}

\section{Different Machine Learning Methods use In Cyber Security REGRESSION}

Regression (or prediction) is easy. Knowledge of the existing data is used to get an idea of the new data. Let's take an example of a house price prediction. In cybersecurity, it can be applied to fraud detection. The characteristics (for example, total amount of suspicious transaction, location, etc.) determine the probability of fraudulent activity. When it comes to the technical aspects of regression, all methods can be divided into two broad categories: machine learning and deep learning. The same is used for other tasks.

\section{CLASSIFICATION}

Sorting is easy too. Suppose you have two stacks of images arranged by type (for example, cats and dogs). In terms of cybersecurity, a spam filter can serve as an example to separate spam from other messages. Spam filters are probably AA's first approach to cybersecurity tasks. Supervised learning perfectly used for classification when examples are known for particular groups. All classes must be defined at the beginning.

\section{CLUSTERING}

The best job for clustering is forensic analysis. The reasons, course and consequences of an incident are in the dark. It is necessary to classify all activities to find anomalies. Malware analysis solutions (i.e., Malware protection or secure email gateways) can implement these to separate legal files from outliers. Another interesting area where clustering can be 
applied is in the analysis of user behaviour. In this case, the application users are grouped together to see if they should belong to a specific group. Clustering is generally not applied to solve a specific cybersecurity task, as it is more one of the subtasks in a pipeline (for example, grouping users into separate groups to adjust risk scores).

\section{ASSOCIATION RULE OF LEARNING}

The best job for clustering is forensic analysis. The reasons, course and consequences of an incident are in the dark. It is necessary to classify all activities to find anomalies. Malware analysis solutions (for example, malware protection or secure email gateways) can implement these to separate legal files from outliers. Another interesting application area for clustering is the analysis of user behaviour. In this case, the application users are grouped to see if they should belong to a particular group. Clustering is generally not used to solve any particular cybersecurity task, as it is more of one of the subtasks in a pipeline (for example, grouping users into separate groups to customize risk assessments).

\section{DIMENSIONALITY REDUCTION}

When it comes to cybersecurity tasks, dimensionality reduction is common in facial recognition solutions, the ones you use on your iPhone.

\section{GENERATIVE METHODS}

The simple job of offensive cybersecurity is to create a list of input parameters to test a particular application for injection vulnerabilities. Alternatively, you can use a web application vulnerability scan tool. One of its modules is testing files for unauthorized access. These tests can mutate existing file names to identify new ones. For example, if a crawler has discovered a file called login.php, it is best to verify the existence of a backup or test your copies by testing names such as login_1.php, login_backup.php, login.php.2017. Generative models are good at it.

\section{MODEL BUILDING}

There are following methods to detect different cyber attacks by Machine Learning are explain below.

1. Botnet Detection

2. Password Security

3. Malware detection 


\section{BOTNET DETECTION}

A bot is software specially designed to perform a task on a regular basis. Therefore, the creation of classification models for the detection of bots is very important both for the research community and for companies.

We have data set from the Kaggle. Botnet detection data set. Data set contains four columns having Ip addresses of different frequency range. By using different machine learning algorithm, we have to detect the Botnet. The detail analysis of algorithm is given below.

First of all, we have to import all the required libraries

import numpy as np

import pandas as pd

from sklearn. Linear_model import*

from sklearn. Tree import *

from sklearn. naive_bayes import *

from sklearn. neighbors import *

from sklearn. metrics import accuracy_score

from sklearn. model_selection import train_test_split

import matplotlib. pyplot as plt

import seaborn as sns

\%Matplotlib inline

load csv fil

data $=$ pd. read_csv('botnetdata.csv')

data.info ()

<class 'pandas. core. Frame. DataFrame'>

Data columns (total 4 columns):

\# Column Non-Null Count Dtype

0 date 20803 non-null object

1 I_ipn 20803 non-null int64

2 r_asn 20803 non-null int64 


\section{3 f 20803 non-null int64}

dtypes: int64(3), object (1)

memory usage: $650.2+\mathrm{KB}$

set the training parameters and test parameters by iloc method

$X=$ data. Iloc $[:[1,3]]$. values

$y=$ data. Iloc $[:-1]$. values

\#Split the dataset into training and testing set by using sklearn

from sklearn. model_selection import train_test_split

X_train, $X \_$test, $y \_$train, $y \_$test $=$train_test_split $(X, y$, random_state $=0$, test_size $=0.2)$

$\cdots$

Here is the thing now we use different algorithm to predict the accurate prediction which model gives accurate result

K-NN algorithm assumes the similarity between the new case/data and available cases and put the new case into the category that is most similar to the available categories.

K-NN algorithm stores all the available data and classifies a new data point based on the similarity. This means when new data appears then it can be easily classified into a well suite category by using K- NN algorithm.

value of $k$ can't be determined so we assume it.

with the help of this algorithm, we can identify the different category and new points in the dataset.

knc $=$ KNeighborsClassifier (n_neighbors $=2$ )

knc.fit (X_train, y_train)

knc_prediction $=$ knc. predict $\left(X \_t e s t\right)$

knc_accuracy $=100.0 *$ accuracy_score (y_test, knc_prediction)

print ("K-Nearest Neighbors accuracy: " + str(knc_accuracy))

K-Nearest Neighbors accuracy: 94.59264599855803 


\section{DESICION TREE}

The goal of using a Decision Tree is to create a training model that can use to predict the class or value of the target variable by learning simple decision rules inferred from prior data (training data).

In Decision Trees, for predicting a class label for a record we start from the root of the tree. We compare the values of the root attribute with the record's attribute. On the basis of comparison, we follow the branch corresponding to that value and jump to the next node.

here the data is in continuous form.

dtc $=$ DecisionTreeClassifier $($ random_state $=0)$

dtc.fit (X_train, y_train)

dtc_prediction $=$ dtc. predict $\left(X \_t e s t\right)$

dtc_accuracy $=100.0 *$ accuracy_score (y_test, dtc_prediction)

print ("Decision Tree Accuracy: " + str(dtc_accuracy))

$\cdots$

Decision Tree Accuracy: 97.26027397260275

Gaussian NB

Gaussian Naive Bayes is a variant of Naive Bayes that follows Gaussian normal distribution and supports continuous data.

Naive Bayes are a group of supervised machine learning classification algorithms based on the Bayes theorem. It is a simple classification technique, but has high functionality. They find use when the dimensionality of the inputs is high. Complex classification problems can also be implemented by using Naive Bayes Classifier.

gnb = GaussianNB ()

gnb.fit (X_train, y_train) 
gnb_prediction $=$ gnb. predict $\left(X_{-}\right.$test $)$

gnb_accuracy $=100.8^{*}$ accuracy_score (y_test, gnb_prediction)

print ("Gaussian Naive Bayes accuracy: " + str(gnb_accuracy))

Gaussian Naive Bayes accuracy: 97.60230713770729

You can analyze the data set form the following pivot plot and index plot data. date.value_counts (). sort_index (). plot ()

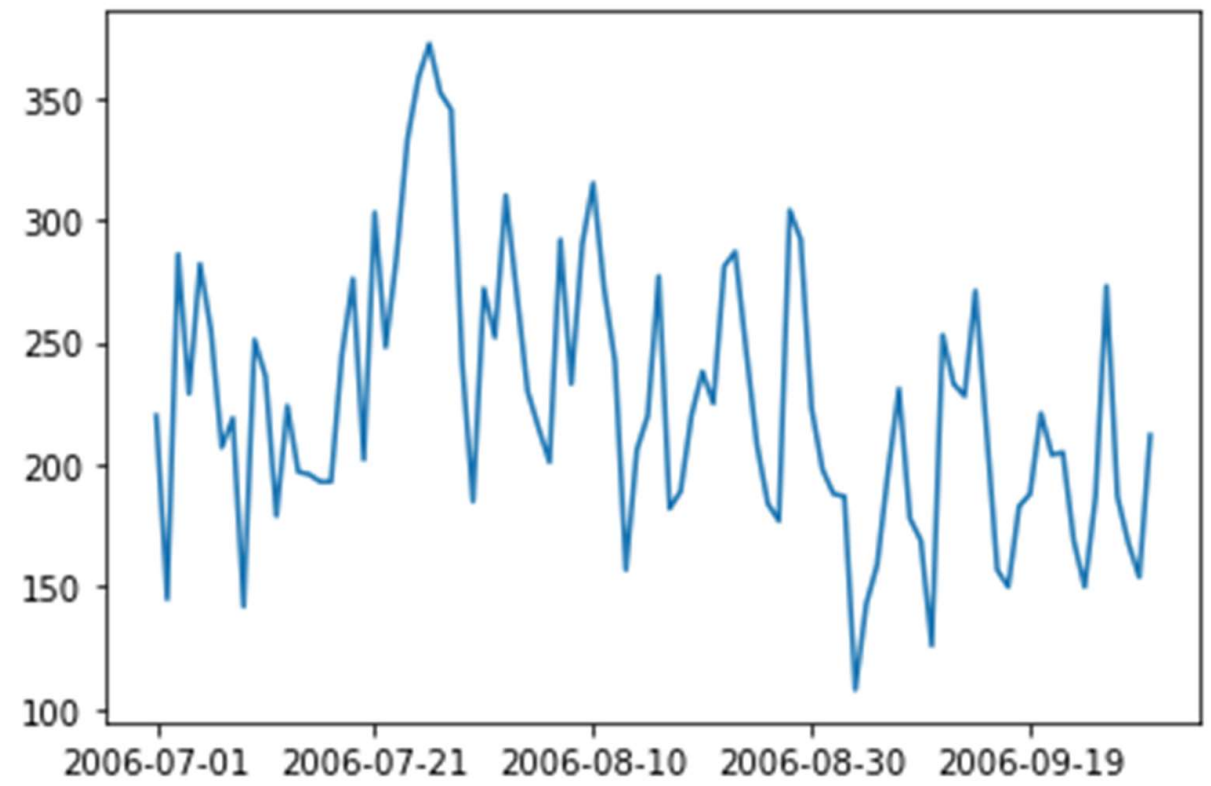


data. pivot_table(['date','I_ipn','r_asn'], ('f')). plot(kind='bar')

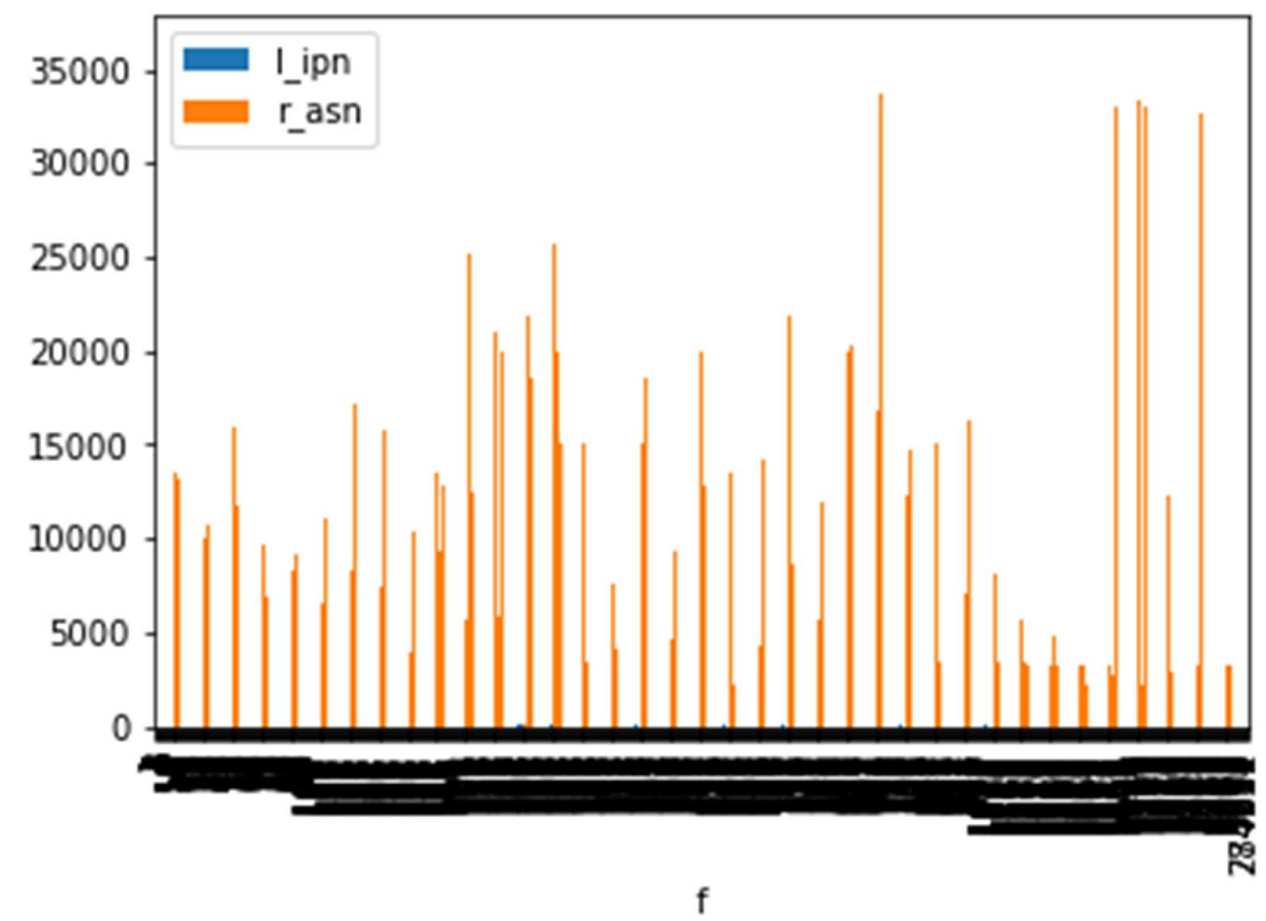

1. Password Security using Machine Learning.

We can use the Machine learning algorithm for password security.

Data explanation and algorithm details

Our data must be read into a DataFrame. This data has two fields: password and password strength.

There are three levels of difficulty for password strength.

1.To make a more robust training, we'll shuffle the data.

2.We divided the DataFrame 80-20.

3.Arrays are used to organize the features and labels.

To tokenize passwords into characters rather than words, we construct a function that divides password strings into characters. The classifier will be able to learn finegrained information about the password dataset as a result of this.

We'll create a pipeline to conduct NLP on the characters in a password, then apply an XGBoost classifier to the results.

We train and test our classifier We choose one of the most common passwords and one that was generated by using a password management system

We can see that the classifier indeed classified the common password as eak (strength 0 ) and the strong password as strong. 
\#Import all required libraries

import numpy as np

import pandas as pd

import matplotlib. pyplot as plt

import seaborn as sns

\# Load the dataset and then describe its features

df = pd. read_csv ('passwordDataset.csv', dtype=\{"password": "str", "strength": "int" , index_col=None)

df. head ()

to check the first five values of the table

df. describe ()

to describe the dataset

df. isnull ()

to check the null value of the data set

sns.set_style('whitegrid')

sns. Countplot ( $x=$ 'strength', data $=d f)$

<AxesSubplot: $x$ label='strength', ylabel='count'> 


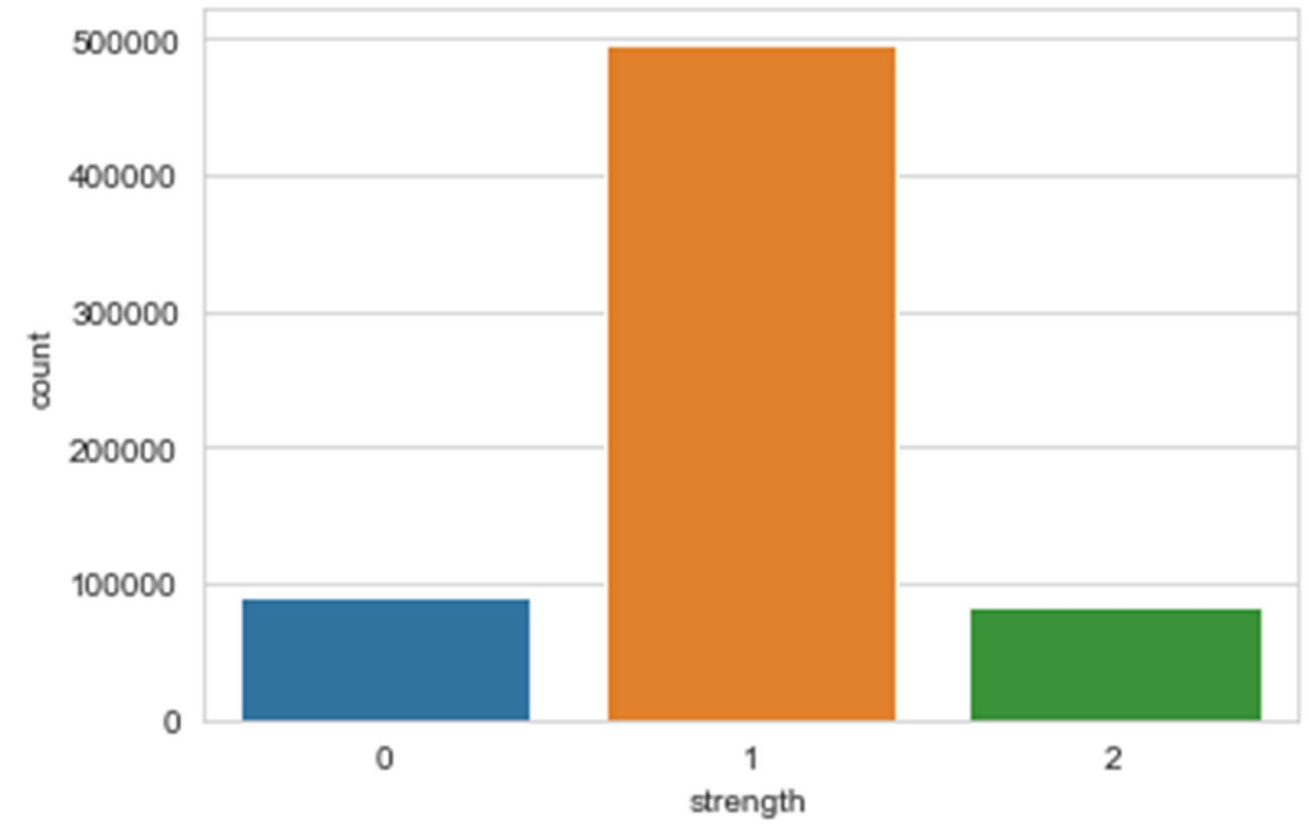

\# Here we shuffle the dataset at random fraction $\mathrm{df}=\mathrm{df}$.sample(frac=1)

\# We split the dataset into traning and test set

I = len (df. index)

train_df $=$ df. Head $($ int $(I * 0.75))$

test_df $=$ df. Tail $($ int $(I * 0.25))$

y_train = train_df.pop("strength"). values

y_test = test_df.pop("strength"). values

X_train $=$ train_df. values. Flatten ()

X_test $=$ test_df. values. flatten ()

\# Created a function that break string into characters

def character_tokens(input_string):

"'"'Break string into characters."'"

return [ $\mathrm{x}$ for $\mathrm{x}$ in input_string]

\section{XGBoost}

Extreme Gradient Boosting is abbreviated as XGBoost. XGBoost is about 10 times quicker than conventional Gradient Boosting due to speed advancements such as parallel computation and cache awareness. XGBoost also provides a split-finding method for optimizing trees, as well 
as built-in regularization to prevent overfitting. In general, XGBoost is a more accurate and quicker variant of Gradient Boosting.

On average, boosting outperforms bagging, and Gradient Boosting is perhaps the best boosting ensemble. XGBoost is undoubtedly the greatest machine learning algorithm since it is an improved version of Gradient Boosting with unrivalled outcomes.

from sklearn. pipeline import Pipeline

from sklearn. feature_extraction.text import TfidfVectorizer

from xgboost import XGBClassifier

password_clf $=$ Pipeline (

[("vect", TfidfVectorizer(tokenizer=character_tokens)), ("clf", XGBClassifier ()),]

)

\#Here we set one variable as random password and one as computer generates password of high entropy

common_password = "qwerty"

strong_computer_generated_password = "c9ICwLBFmdLbG6iWla4H"

password_clf.fit (X_train, y_train)

password_clf. score (X_test, y_test)

$91 \%$

password_clf. Predict ([common_password, strong_computer_generated_password])

\section{Malware protection}

We acquire a pool of clean samples and a pool of ransomware samples from the internet in our attempt to develop a machine learning-based ransomware classifier. With the aid of an attribute extractor [3] script, the attributes of PE files are extracted from these samples, and a dataset is created. After that, the dataset is divided into training and test sets. The training set is then used to train a supervised machine learning model, which is subsequently tested on the test set.

With the ever-expanding threat landscape, it's becoming increasingly difficult to keep up with the rate at which new threats are produced and disseminated. Not only are there new dangers, but malware developers are as well.

In most cases, manual analysis includes a signature-based detection. As a result, until the threat is manually evaluated and an anti-virus signature is provided through a definition 
update, some individuals are constantly afflicted and suffer from a new danger. As a result, traditional signature-based detection is useless in the face of zero-day assaults. As a result, consumers who are subjected to ransomware assaults from new threats are unprotected by signature-based solutions.

In most situations, signature-based detection is included in manual analysis.

As a result, until the threat is personally assessed and an anti-virus signature is supplied via a definition update, some people are continually infected and exposed to new threats. As a result, in the face of zero-day attacks, traditional signature-based detection is rendered ineffective. As a result, users who are targeted by new ransomware attacks are left vulnerable to signature-based solutions.

\section{MALWARE DETECTION USING ISOLATION-FOREST}

IsolationForests (IF), like Random Forests, are construct dependent on choice trees. Furthermore, since there are no pre-characterized marks here, it is an unaided model. IsolationForests were assembled dependent on the way that irregularities are the information focuses that are "few and unique". In an Isolation Forest, arbitrarily sub-tested information is handled in a tree structure dependent on haphazardly chose highlights. The examples that movement more profound into the tree are less inclined to be irregularities as they required more slices to separate them. Additionally, the examples which end up in more limited branches demonstrate irregularities as it was simpler for the tree to isolate them from different perceptions.

\section{HOW ISOLATION FOREST WORK}

At the point when given a dataset, an arbitrary sub-example of the information is chosen and doled out to a paired tree. Fanning of the tree begins by choosing an arbitrary component (from the arrangement of all $\mathrm{N}$ highlights) first. And afterward expanding is done on an arbitrary limit (any worth in the scope of least and most extreme upsides of the chose include). In the event that the worth of an information point is not exactly the chosen limit, it goes to the left branch else to the right. Also, along these lines a tree is parted into left and right branches.

This cycle from stage 2 is proceeded recursively till every information point is totally secluded or work max depth (if characterized) is reached.

\#lmport all required libriries

import pandas as pd

import numpy as np

from sklearn import*

import matplotlib. pyplot as plt

from sklearn. Metrics import accuracy_score 
random_seed $=$ np. random. RandomState (12)

$\mathrm{df}=$ pd. read_csv ("malware dataset.csv")

df. head ()

we use this command to get the first five value of the datasets

df. shape

to know the shape of the dataset

df. isnull (). sum ()

this command uses to know the null value in the data set

df1['classification'] = df1.classification.map (\{'benign':0, 'malware':1\})

. map function uses to assign the value to the categorical data

After that we check the shape of the new dataset by

df. shape

then check the dimension

df. ndim

after that

we analyze the data using the countplot

import seaborn as sns

sns. countplot(df1["classification"]) 


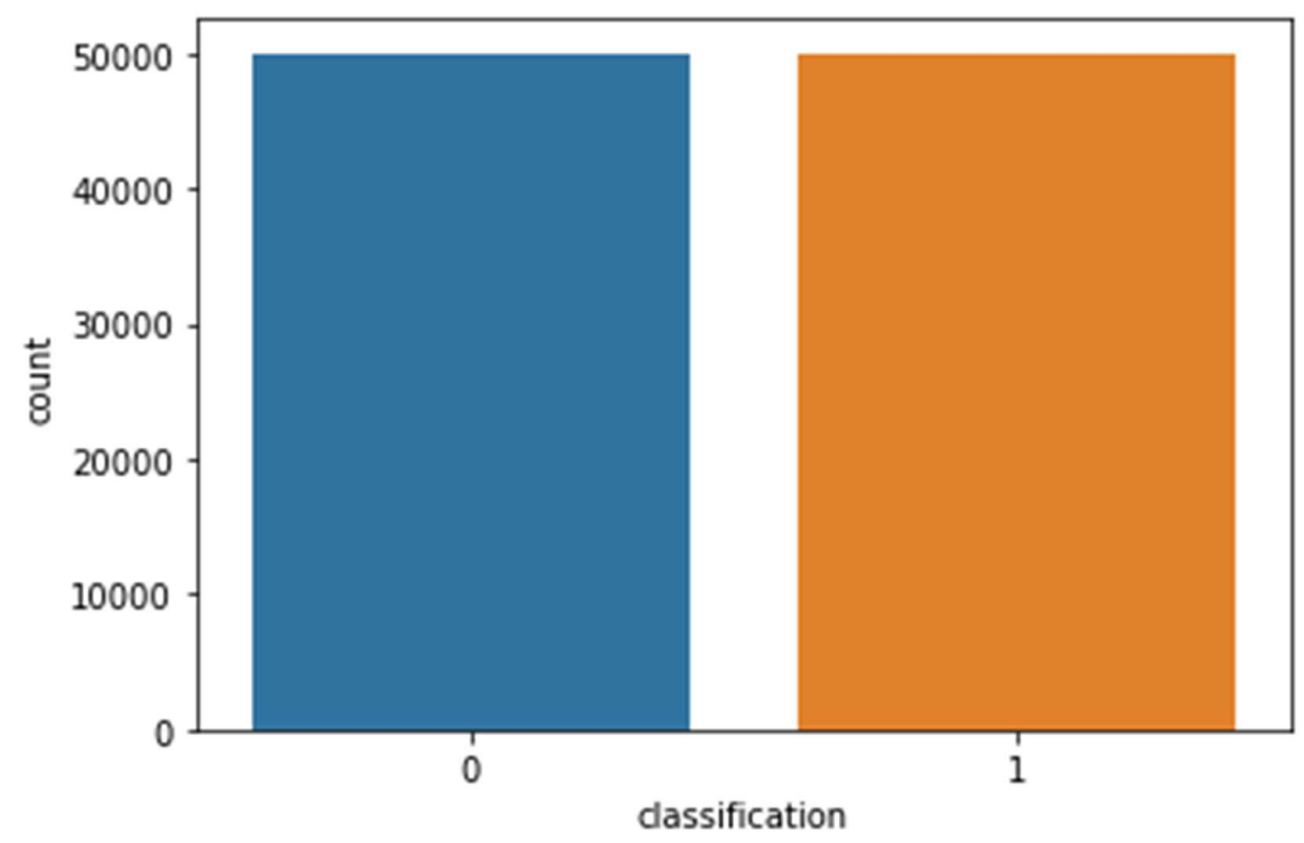

Now assign $X$ and $y$ values

X=df1.drop(["hash","classification",'vm_truncate_count','shared_vm','exec_vm','nvcsw','maj _flt','utime'], axis=1)

y=df1[["classification","hash"]]

now

split dataset into the train and test datasets

from sklearn. model_selection import train_test_split

$X \_$train, $X \_$test, $y \_$train, $y \_$test $=$train_test_split $(X, y$, random_state $=0$, test_size $=0.2)$

Now fit the isolation forest into the training and test set

from sklearn. ensemble import IsolationForest

$\mathrm{clf}=$ IsolationForest ()

clf. Fit(X_train)

Y_pred_train $=$ clf. Predict $\left(X \_t r a i n\right)$

y_pred_test $=$ clf. predict $\left(X \_t e s t\right)$ 
$X=$ range $(500)$

$y=$ range $(500)+n p$. random. RandomState $(0,300,500)$

plt. Scatter $(X, y)$

plt. rcParams.update (\{'figure. fig size':(10,8), 'figure.dpi':100\})

plt. Title ('Simple Scatter plot')

plt. Xlabel ('X - value')

plt. Ylabel ('Y - value')

plt. show ()

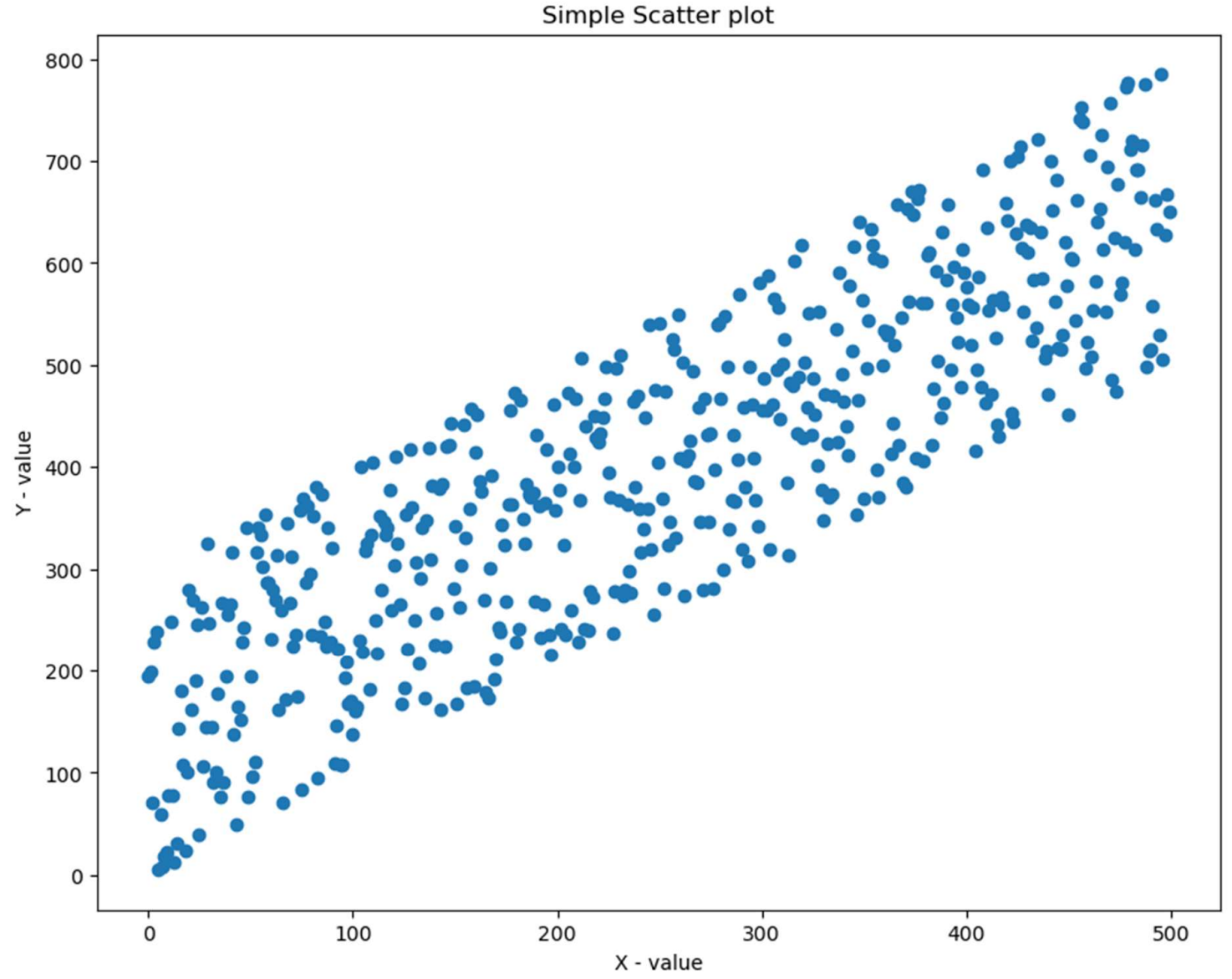

This is the malware detection using the IsolationForest.

Similarly, we can use time series analysis to determine the type of malware by its name.

We can use the convolutional neural network and artificial neural network to detect the malware properties. 
For the following types detection, we can use different machine learning algorithm.

\section{Clustering for Spam detection}

A Spam email filter, for example, can differentiate unsolicited mail from ordinary messages in phrases of cybersecurity. Spam filters have been arguably the first device studying strategies to cybersecurity. Forensic evaluation is the precise mission for clustering in cyber safety. An incident's cause, course, and consequences continue to be unknown. It is important to categorise a study. (Semi-e-mail gateways or virus prevention, for example). It's viable to use it to documents that are not outliers. Clustering is usually now no longer used to resolve a selected undertaking in cyber safety in view that it's far greater want to the only of a fabric withinside the pipeline.

\section{Machine Learning for Industry}

cyber safety This precept is especially relevant to incident reaction. If a corporation is hit with the aid of using a wave of incidents and has a range of response options, the device will study which kind of reaction to apply for every occurrence.

\section{Dimensionality reduction to handle large amount of unlabelled data}

If you are managing a complex device with unlabelled records and a lot of viable features, dimensionality reduction or generalizations comments are simply as crucial as classification. When it involves cybersecurity jobs, dimensionality reduction is usually utilized in facial detection systems, inclusive of those discovered to your phone.

\section{Generative method for prediction}

The mission of generative method isn't the same as that of the previously defined strategies. generative fashions are intended to stimulate real records based on beyond records, while different jobs cope with current statistics and associated judgments.

\section{CONCLUSION}

Machine learning can be successfully used for developing authentication structures, comparing the protocol implementation, assessing the safety of human interaction proofs, smart meter data profiling, etc. Although device studying facilitates preserving numerous structures safe, the device studying classifiers themselves are vulnerable to malicious attacks. There have been a few paintings directed to enhancing the effectiveness of device studying algorithms and protective them from numerous attacks. There are many possibilities in records safety to use device studying to address numerous challenges in such complex domain. Spam detection, virus detection, and surveillance. In the sector of Al-pushed cybersecurity, the idea of Al-primarily based totally protection intelligence modeling mentioned on this paper can assist the cybersecurity computing technique to be greater actionable and intelligent. Based on our study, we've additionally highlighted numerous studies troubles and capability instructions that may assist researchers do destiny studies withinside the area. Overall, we trust this paper may be served as a reference 


\section{REFERENCES}

Li S, Da Li X, Zhao S. The internet of things: a survey. Inf Syst Front. 2015;17(2):243-59.

2. Velte T, Velte A, Elsenpeter R. Cloud computing, a practical approach. New York: McGrawHill Inc; 2009.

3. Sarker IH, Kayes ASM, Badsha S, Alqahtani H, Watters P, Ng A. Cybersecurity data science: an overview from machine learning perspective. J Big Data. 2020;7(1):1-29.

4. Ibm security report. https://www.ibm.com/security/data-breach. Accessed 20 Oct 2019.

5. Fischer EA. Cybersecurity issues and challenges: in brief. 2014. 6. Anwar S, Mohamad Zain J, Zolile MF, Inayat Z, Khan S, Anthony B, Chang V. From intrusion detection to an intrusion response system: fundamentals, requirements, and future directions. Algorithms. 2017;39(2):10.

6. Joshua Saxe, Konstantin Berlin, "Deep Neural Network Based Malware Detection Using Two-Dimensional Binary Program Features," IEEE 2015 10th International Conference on Malicious and Unwanted Software: "Know Your Enemy".

7. Source 1 for Ransomware and Non-Ransomware Samples [Online] - www.malshare.com.

8.Source 2 for Ransomware and Non-Ransomware Samples [Online] https://github.com/ytisf/theZoo

9. Json Brown Lee "Feature Selection for Machine Learning in Python". [Online] http://www.ieee.org/

10.Tianqi Chen and Carlos Gastrin "XGBoost: A Scalable Tree Boosting System" [Online]. https://arxiv.org/pdf/1603.02754.pdf

11. Complete Guide to Parameter Tuning in XGBoost (with codes in Python)

12. [Online] https://www.analyticsvidhya.com/blog/2016/03/completeguide-parametertuning-xgboost-with-codes-python/

[Online]https://www.stat.berkeley.edu/ breiman/randomforest2001.pdf

13. Mohammadi S, Mirvaziri H, Ghazizadeh-Ahsaee $M$, Karimipour H. Cyber intrusion detection by combined feature selection algorithm. J Inf Secur Appl. 2019; 44:80-8.

14.Tapiador JE, Orfila A, Ribagorda A, Ramos B. Key-recovery attacks on kids, a keyed anomaly detection system. IEEE Trans Dependable Secur Comput. 2013;12(3):312-25.

15. Tavallaee M, Stakhanova N, Ghorbani AA. Toward credible evaluation of anomaly-based intrusion-detection methods. IEEE Trans Syst Man Cybern Part C (Appl Rev). 2010;40(5):516-

16. Foroughi F, Luksch P. Data science methodology for cybersecurity projects. arXiv preprint arXiv:1803.04219. 2018.

17. Saxe J, Sanders H. Malware data science: attack detection and attribution. 2018.

18. Rainie L, Anderson J, Connolly J. Cyber-attacks likely to increase. Digit Life. 2014;2025. 
19. National Research Council et al. Toward a safer and more secure cyberspace. 2007.

20.. Kaggle datasets

21. sklearnAPIs

22. Udemy

23. towardsdatascience.com

24. SN computer science (2021) 